\section{Our approach to squamous intraepithelial lesions of the uterine cervix}

\section{Alexandra N Kalof, Kumarasen Cooper}

\section{Morphological analysis remains the "gold standard" in the diagnosis and grading of $\mathrm{CIN}$}

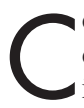
rvical carcinoma is a significant contributor to cancer-related morbidity and mortality worldwide and the role of human papillomavirus (HPV) in the development of preinvasive and invasive cervical lesions is well established. ${ }^{12}$ Although significant advances have been made in elucidating the potential mechanisms of cellular transformation by HPV and in the molecular detection of HPV in cytological and surgical specimens, morphological assessment of surgical material remains the "gold standard" in the diagnosis of cervical intraepithelial neoplasia (CIN). Although management of preinvasive cervical disease depends on many factors including the age of the patient, parity and size of the lesion, clinical management often requires confirmation of CIN by histological examination with subsequent surgical treatment of highgrade lesions (CIN 2 or CIN 3). This has fueled attempts at more objective, reproducible diagnostic parameters to accurately diagnose CIN. The histological features of preinvasive cervical neoplasia (CIN 2 and 3) are well understood, however inconsistent use and misinterpretation of the morphological criteria could lead to significant intraobserver and interobserver variability. ${ }^{3-5}$ This lack of reproducibility and the fact that there are many benign changes that can mimic dysplasia of the cervical epithelium (eg, cervical atrophy and immature squamous metaplasia) have led to significant efforts to identify a surrogate marker for high-grade CIN. In the following discussion, we will present the criteria that we use in our general surgical pathology practice, along with potential pitfalls and approaches to histological mimics of cervical neoplasia. We will propose incorporating the use of ancillary techniques such as immunohistochemistry for $\mathrm{pl}^{\mathrm{INK} 4 \mathrm{a}}$ and MIB-1 (Ki-67), as well as the role of HPV in situ hybridisation, in the grading of CIN.

\section{MORPHOLOGICAL DIAGNOSIS OF CIN}

In our general surgical pathology practice at the University of Vermont, Fletcher
Allen Health Care, Burlington, Vermont, USA, we see approximately 30000 surgical cases a year, 3.3\% of which are colposcopically guided cervical biopsies. Each cervical biopsy, initiated by an abnormal Papanicolaou smear with a diagnosis of low-grade squamous intraepithelial lesion (LG-SIL), high-grade squamous intraepithelial lesion (HGSIL) or atypical squamous cells of undetermined significance, which cannot rule out HG-SIL, is reviewed in conjunction with the referring Papanicolao smear. Deeper levels are performed on the paraffin block if a lesion is not identified on the corresponding biopsy specimen.

\section{General classification of CIN}

A diagnosis of CIN is based primarily on the presence of nuclear atypia and loss of normal squamous maturation (polarity). Accurate grading of CIN lesions becomes important as we begin to understand the rates of regression, persistence and progression of the low-grade (CIN 1) and high-grade lesions (CIN 2 and 3), as their treatment and clinical follow-up algorithms are quite different. ${ }^{6}$

We have adopted a two-tiered approach for reporting cervical cytological specimens, adapted from the Bethesda system introduced in the late 1980s, and extrapolated this system for reporting our histopathological specimens. This system divides non-invasive cervical squamous epithelial lesions into LG-SIL and HG-SIL. This terminology reflects the natural history of HPV infection, and differentiates cervical squamous lesions associated with productive, acute HPV infection in which the virus remains in an episomal physical state (LG-SIL) from those squamous lesions resulting from transformation to a proliferative HPV infection in which the virus is integrated into the host genome (HG-SIL). ${ }^{7}$ We have retained the CIN terminology within the two-tiered system, and include CIN 1 in LG-SIL (including lesions such as condyloma accuminatum and flat condyloma) and CIN 2/CIN 3 into HG-SIL. Low-grade lesions (CIN 1) have been shown to have a high rate of spontaneous regression within 1 year, ${ }^{8}$ despite the fact that most of these lesions $(>80 \%)$ contain high-risk (HR) HPV types. ${ }^{79}$ Concordantly, lowgrade lesions show a relatively low rate of progression to higher grades of CIN $(\sim 10 \%)$ and invasion $(\sim 1 \%){ }^{8}$ In contrast, high-grade lesions such as CIN 3 show a $12 \%$ risk of progression to invasion. ${ }^{8}$ Hence the two-tiered approach for reporting cervical dysplasia introduced by the Bethesda system supports the twotiered approach to clinical management, which generally includes conservative clinical management of LG-SIL (CIN 1) and referral to cervical cone or loop electrosurgical excision procedure for high-grade lesions (CIN 2 and 3).

\section{Mechanisms of carcinogenesis}

Although the likelihood of progression clearly increases with increasing grade of cervical intraepithelial neoplasia, a proportion of high-grade lesions could still regress. Persistent HPV infection with HR subtypes has been shown to be a risk factor for persistent and/or progressive cervical dysplasia. ${ }^{10}$ It has also been proposed that HPV DNA integration into host DNA is critical in cervical carcinogenesis $^{11}{ }^{12}$ through disruption of the El/ E2 open reading frames of HPV genome and subsequent loss of the E2-controlled regulation of $\mathrm{E} 6$ and $\mathrm{E} 7,{ }^{12}$ the viral oncogenes of HPV. Through inactivation of the host p53 and pRb proteins, uncontrolled E6 and E7 expression in proliferating basal and parabasal cells results in loss of the normal maturation sequence, representing persistent, proliferative HPV infection. This cellular immortalisation presumably results in the transformation into high-grade dysplasia (CIN 2 and 3), with a potential to progress to invasive carcinoma.

\section{Diagnosis of low-grade squamous intraepithelial lesions (CIN 1)}

Morphological features associated with productive HPV infection include koilocytosis, dyskeratosis and cytonuclear abnormalities such as multinucleation, nuclear hyperchromasia and irregular nuclear contours. In contrast with HGSIL, the overall pattern of squamous maturation is preserved and mitotic figures are restricted to the level of the basal/ parabasal cells, the level at which cellular replication normally occurs. Although the cytonuclear abnormalities typically involve the entire thickness of the epithelium (hence, accessible by cytologic smear), there is a retention of squamous maturation and most diagnostic abnormalities are present in the lower one-third of the epithelium. The minimum criteria 
A

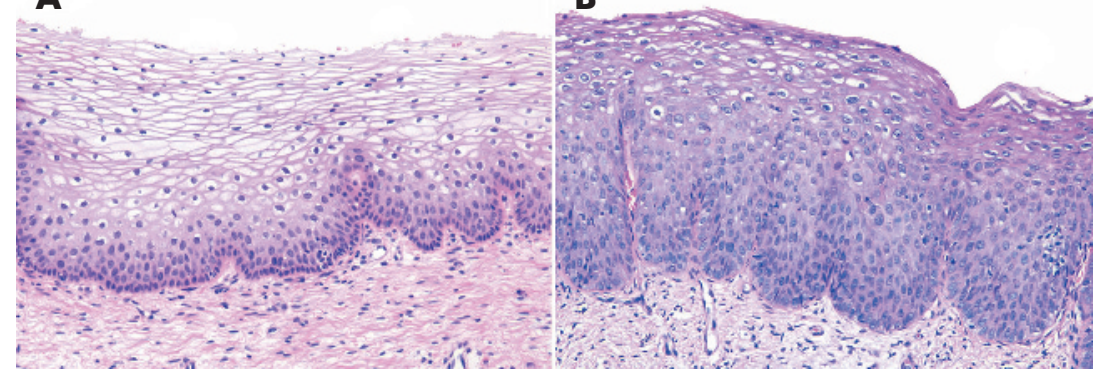

Figure 1 (A) Normally glycogenated squamous epithelium. (B) Low-grade squamous intraepithelial lesion (LG-SIL) exhibiting true koilocytosis and cytonuclear atypia.

necessary for a diagnosis of LG-SIL are poorly defined. As heavily glycogenated squamous epithelium (fig 1A) and reactive epithelium with inflammation can closely mimic true koilocytosis, ${ }^{13}$ we require a combination of koilocytosis (perinuclear cytoplasmic clearing with peripheral condensation of the cytoplasm) and significant cytonuclear changes such as irregular nuclear contours, binucleation/multinucleation, hyperchromasia and/or cellular pleomorphism to make a diagnosis of lowgrade dysplasia (CIN l; fig lB).

Diagnosis of high-grade squamous intraepithelial lesions (CIN 2 and 3 ) Morphologically, high-grade dysplasia (CIN 2 and 3 ) is characterised by a loss of upward maturation, nuclear crowding, loss of nuclear polarity and significant cytonuclear atypia. The cytonuclear atypia includes increased nuclear-to-cytoplasmic $(\mathrm{N}: \mathrm{C})$ ratios, irregular nuclear contours and coarse chromatin. Importantly, increased proliferation is seen with mitotic figures sometimes identified in the upper one-half of the epithelium, some of which could be atypical. In CIN 2, it is generally full-thickness cellular atypia; however, there is a persistence of squamous maturation with stratification at the superficial aspect of the epithelial surface. Most cytonuclear abnormalities, however, are present in the lower and middle-third of the epithelium (fig 2A). By contrast, the changes in CIN 3 are more uniform with full-thickness cytonuclear atypia and minimal to absent maturation (fig 2C). Mitotic figures are
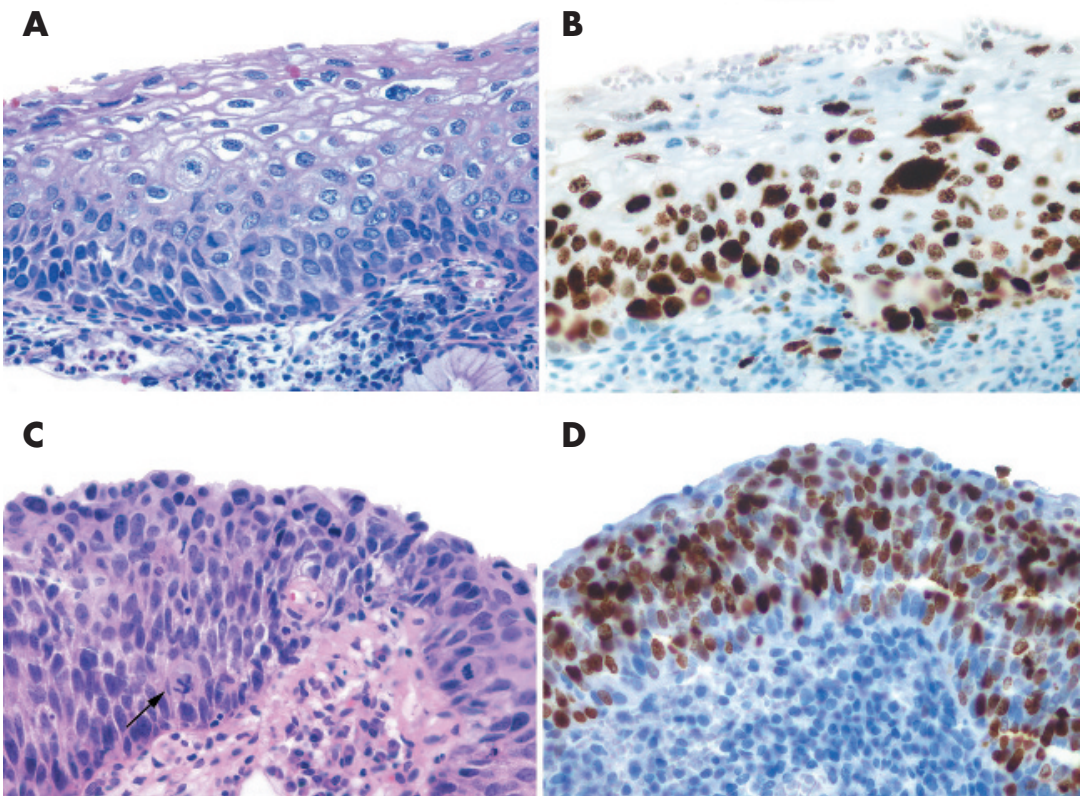

D

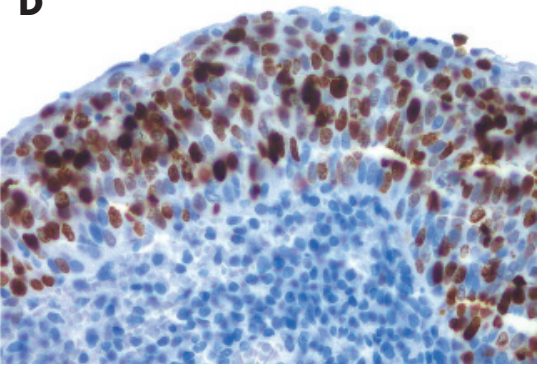

Figure 2 High-grade squamous intraepithelial lesion (HG-SIL). Cervical intraepithelial neoplasia 2 (CIN2): (A) H\&E-stained section demonstrating mitotic figures located in the upper one-half of the epithelium; (B) Ki-67 immunohistochemistry demonstrating nuclear positivity in the upper two-thirds of the epithelium. CIN 3: (C) H\&E-stained section demonstrating mitotic figures at the most superficial aspect of the epithelium, including an atypical form (arrow); (D) Ki-67 immunohistochemistry demonstrating full-thickness nuclear positivity. generally numerous, and can extend to the superficial aspects of the epithelium.

\section{Important mimics of HG-SILs}

The main differential diagnoses of HGSILs include reactive/inflammatory changes, basal-cell hyperplasia, immature squamous metaplasia, squamous atrophy and LG-SIL. The diagnostic challenges are greater when such conditions are superimposed onto HPV-associated changes.

\section{Basal-cell hyperplasia versus HG-}

SIL

Basal-cell hyperplasia is characterised by thickening of the basal and parabasal zones with associated nuclear enlargement and cytoplasmic basophilia. ${ }^{14}$ Above this thickened cell layer, squamous maturation and polarity is retained with a normally glycogenated squamous epithelium. Although the "picket fence" arrangement of the basal cells is lost, the cells retain oval nuclear contours without nuclear pleomorphism or hyperchromasia (fig 3). ${ }^{14}$

\section{Immature squamous metaplasia} versus HG-SIL

Squamous metaplasia, a normal physiological process, is encountered frequently in cervical biopsies and loop electrosurgical excision procedures. The varied morphology results from the different stages of squamous metaplasia. Distinguishing between immature squamous metaplasia and HG-SIL can be extremely difficult, given the presence of increased $\mathrm{N}$ :C ratio and a relative lack of squamous maturation in both lesions. The metaplastic cells are generally uniform, round to oval, with a single nucleolus (fig 4A-C). The nuclear contours are generally smooth. The presence of significant cellular crowding, nuclear atypia and increased mitotic figures in the upper half of the epithelium can be the most helpful morphological features in supporting a diagnosis of

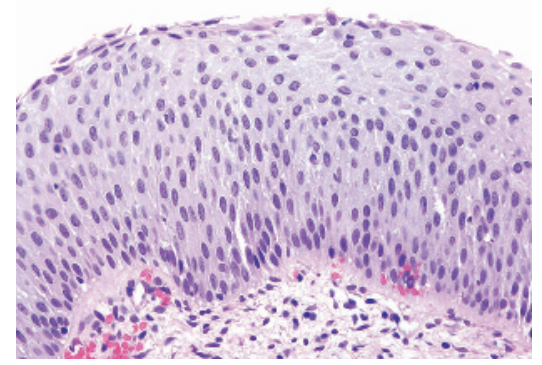

Figure 3 Basal-cell hyperplasia. H\&E-stained section demonstrating loss of the "picket fence" arrangement of the basal-cell layer and the near full-thickness proliferation of monomorphic, basaloid cells without nuclear atypia or hyperchromasia. 


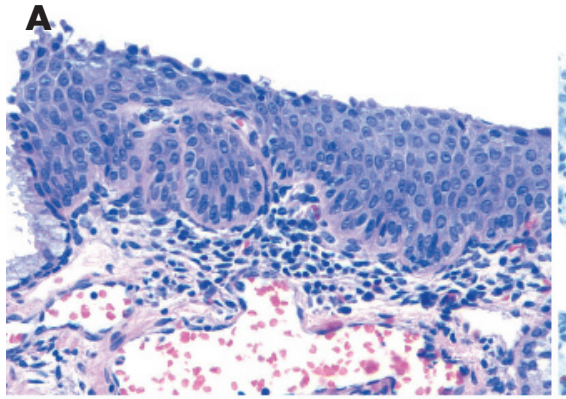

D

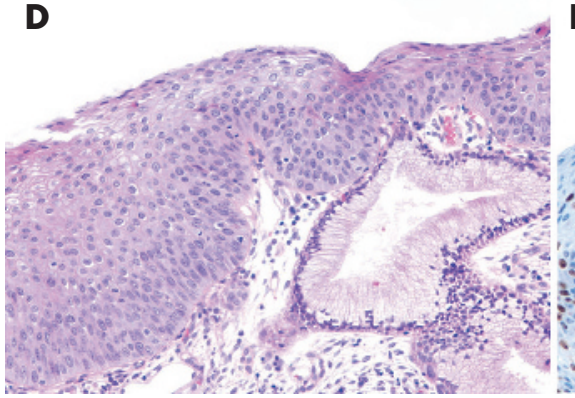

B

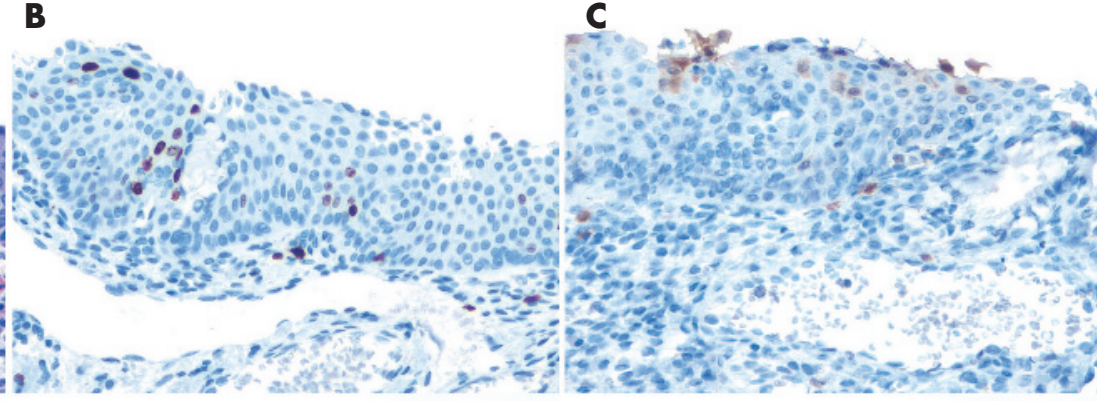

$\mathbf{E}$

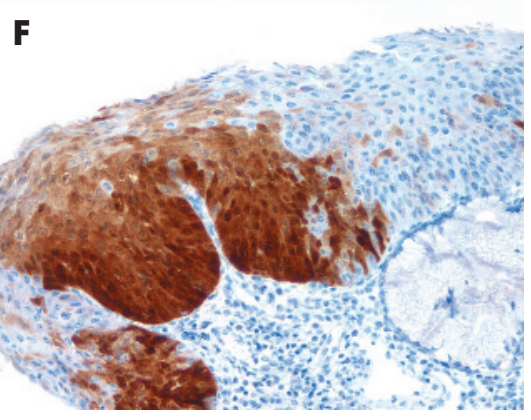

Figure 4 Immature squamous metaplasia: (A-C) H\&E-stained section; (B) Ki-67 immunohistochemistry demonstrating immunoreactivity restricted to the basal/parabasal zone; (C) pl6 $6^{\mathbb{N N} 4 a}$ immunohistochemistry demonstrating non-specific, cytoplasmic blush. High-grade squamous intraepithelial lesion (HG-SIL): (D-F) H\&E-stained section; (E) Ki-67 immunohistochemistry demonstrating immunoreactivity in the upper two-thirds of the epithelium; (F) p16 ${ }^{\text {INK4a }}$ immunohistochemistry demonstrating strong, full-thickness nuclear and cyłoplasmic immunoreactivity.

high-grade CIN (fig 4D-F). CIN can show extensive involvement of endocervical crypts and the presence of significant nuclear pleomorphism should initiate a diagnosis of dysplasia, despite the presence of a superficial layer of endocervical glandular cells. ${ }^{15}$

Reactive atypia versus HG-SIL Reactive and reparative epithelial changes are commonly seen in cervical biopsy specimens and are characterised by enlarged, vesicular nuclei and prominent nucleoli. There is associated inflammation, either acute or chronic, and an increase in mitotic activity is often observed. Difficulty arises when these reactive changes are superimposed onto CIN. The presence of coarse, clumped nuclear chromatin and nuclear contour irregularities suggest possible underlying dysplasia. The atypia seen in purely reactive conditions is usually restricted to the basal half of the epithelium, ${ }^{16}$ with some maintenance of squamous maturation at the epithelial surface. In difficult cases that exhibit reactive-type changes combined with significant cytonuclear atypia (eg, clumped chromatin and irregular nuclear contours), we use $\mathrm{pl}^{\text {INK4a }}$ immunohistochemistry as a surrogate marker for cervical dysplasia, with strong, diffuse nuclear and cytoplasmic staining indicating the presence of squamous dysplasia (fig 5).

Squamous atrophy versus HG-SIL Squamous atrophy is characterised by thinning of the cervical squamous epithelium without evidence of cellular differentiation. Atrophy occurs in states of low oestrogen such as menopause or in women taking oral contraceptives with low levels of oestrogen. ${ }^{15}$ In squamous atrophy, the cells are predominately of the parabasal type, which can appear uniformly hyperchromatic and monomorphic (fig 6). The presence of increased $\mathrm{N}: \mathrm{C}$ ratios in squamous atrophy and the lack of normal maturation serve as potential mimics of high-grade CIN. Nuclear pleomorphism, however, will be lacking unless there are superimposed reactive changes, in which case the atypia is generally restricted to the lower half of the epithelium. ${ }^{16}$ Low to absent mitotic activity is invaluable to the diagnosis of squamous atrophy.

\section{LG-SIL versus HG-SIL}

The morphological distinction between LG-SIL and HG-SIL can be difficult if the cytonuclear abnormalities are in full
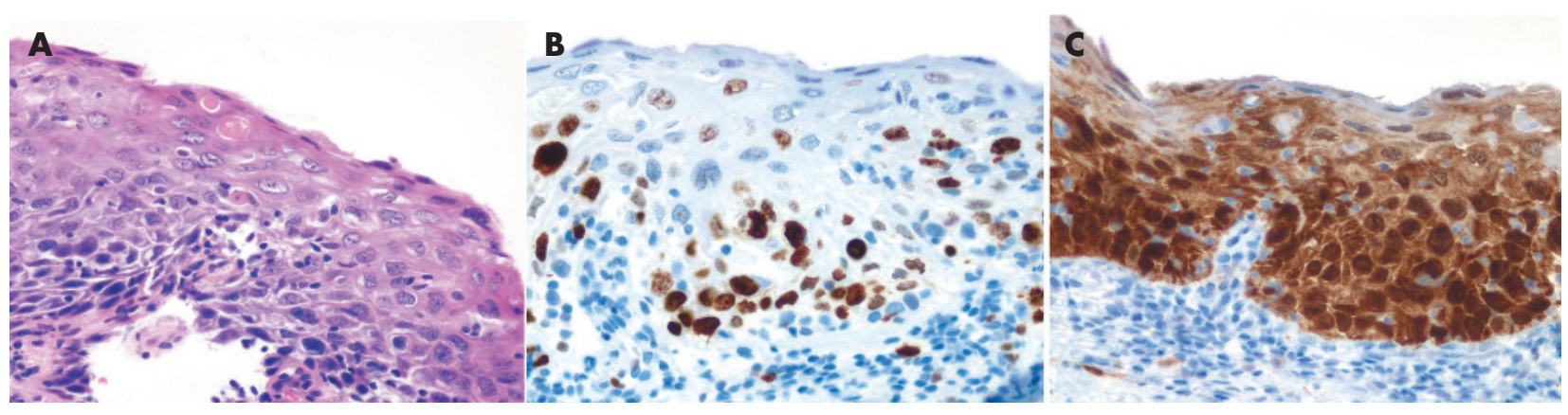

Figure 5 High-grade squamous intraepithelial lesion (HG-SIL) with superimposed reactive changes: (A) H\&E-stained section; (B) full-thickness nuclear immunohistochemical staining for Ki-67; (C) two-thirds to full-thickness immunohistochemical staining for pl $6^{\text {INK4a. }}$. 
A

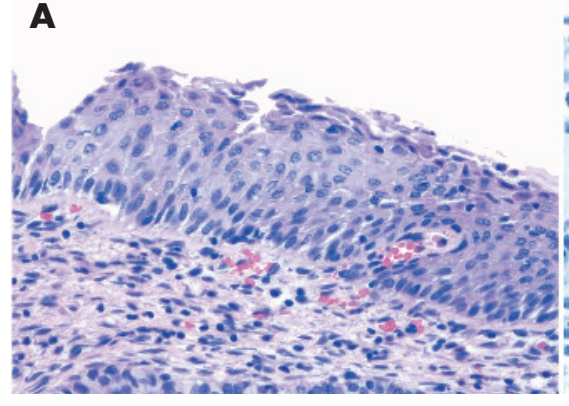

B

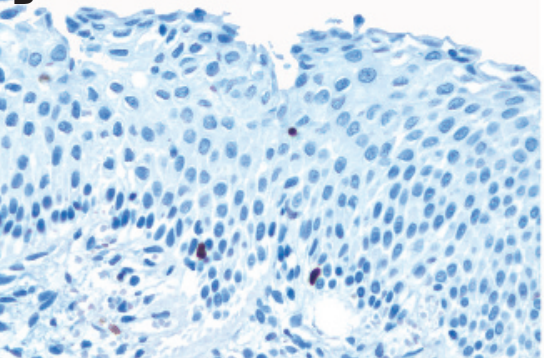

C

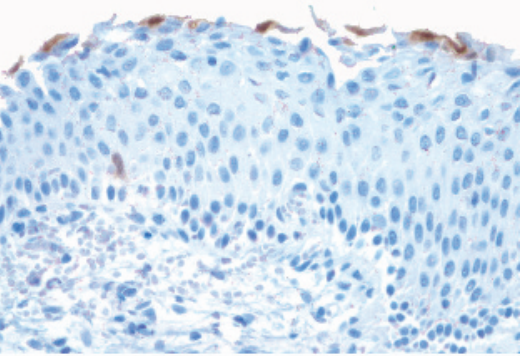

Figure 6 Squamous atrophy: (A) H\&E-stained section showing monomorphic, hyperchromatic basal cells lacking normal maturation; (B) Ki-67 immunohistochemistry exhibiting focal positivity restricted to the basal/parabasal cell layer (note small lymphocyte within the epithelium displaying nuclear positivity); (C) pl $6^{\mathbb{N K K} 4 a}$ immunohistochemistry highlighting residual normal endocervical glands at the most superficial aspect. The squamous cells are p $16^{\text {INK } 4 a^{\prime}}$ negative.

thickness and mitotic activity approaches the mid-third of the epithelial surface. As mentioned previously, the histological changes in LG-SIL are generally concentrated in the lower one-third of the epithelium. From low-power microscopic observation, we assess the cellularity of the lesion, and mitotic figures are looked for on high power, especially atypical forms. If mitotic figures are present above the basal third of the epithelium, our suspicion for HG-SIL is enhanced. LG-SIL could exhibit marked cytonuclear abnormalities extending to the epithelial surface; however, the $\mathrm{N}: \mathrm{C}$ ratio is generally not significantly increased, as is seen in HG-SIL. As cervical dysplasia represents a morphological continuum, we appreciate that some lesions border LG-SIL (CIN 1) and HG-SIL (CIN 2 and $3)$. In these cases, we will employ the use of ancillary techniques such as $\mathrm{pl}^{\mathrm{INK} 4 \mathrm{a}}$ and Ki-67 to supplement the morphological interpretation. If a lesion displays morphological characteristics that are intermediate between CIN 1 and CIN 2 (fig 7A), we will make a diagnosis of HGSIL if Ki-67 highlights nuclear positivity extending into the upper third of the epithelium (fig 7B) and $\mathrm{pl}^{\mathrm{INK} 4 \mathrm{a}}$ displays two-thirds to full-thickness nuclear and cytoplasmic immunostaining of the epithelium (fig 7C).

\section{HG-SIL versus invasive squamous- cell carcinoma}

Evaluation of possible invasion can be extremely difficult in cervical biopsies that exhibit morphological features of highgrade CIN without a significant amount of subepithelial stroma, especially if the biopsy is tangentially cut or maloriented. The presence of paradoxical maturation at the base of the epithelium, including large, atypical keratinised cells and keratin pearls, can raise the suspicion for invasive disease. In a comparative study of the morphological features of in situ versus invasive carcinoma, Leung et $l^{17}$ found that the presence of bizarre giant cells up to five times the size of basal cells, the presence of keratinised cells or keratin pearls, necrosis (often comedo-like) and neovascularisation are histological features associated with invasive carcinoma. If a definitive diagnosis cannot be made with certainty, we use the diagnosis of "HG-SIL (CIN 3), cannot exclude invasive carcinoma". The distinction rests with these important morphological features, as the immunohistochemical profiles of CIN 3 and invasive carcinoma are very similar.

\section{ANCILLARY STUDIES AND SURROGATE MARKERS OF HG-SIL p $16^{\text {INK4a }}$}

The value of $\mathrm{p} 16^{\mathrm{INK} 4 \mathrm{a}}$ as a surrogate marker of HR-HPVs and CIN has been well established in recent years, with studies showing increased immunoexpression of pl6 ${ }^{\text {INK4a }}$ in neoplastic cervical epithelial cells and a positive correlation with HR HPV infection and the degree of cervical neoplasia. ${ }^{18-24}$ The pl6 gene product normally acts to inhibit progression through the cell cycle by binding to cyclin-dependent kinase 4/6, thereby preventing the phosphorylation and subsequent inactivation of the retinoblastoma $(\mathrm{Rb})$ gene product. The depletion of E2F transcription factor decelerates the cell cycle. Although loss of functional pl6 and decreased pl6 protein immunoreactivity has been associated with carcinogenesis in a variety of organ systems, ${ }^{25-30}$ cervical dysplasia and carcinoma are characterised by increased levels of pl6 protein immunoexpression. The paradoxical overexpression of $\mathrm{p} 16^{\mathrm{INK} 4 \mathrm{a}}$ is probably secondary to its involvement in a negative feedback loop with the $\mathrm{Rb}$ protein. As $\mathrm{Rb}$ function is reduced through inactivation by HPV E7, immunoexpression of p $16^{\text {INK4a }}$ is enhanced..$^{31} \mathrm{pl} 6^{\mathrm{INK} 4 \mathrm{a}}$ is therefore linked to HPV infection through
A

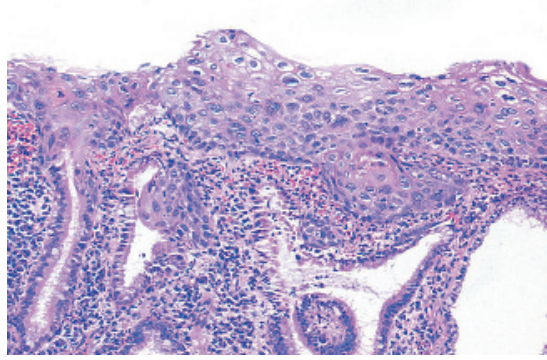

B

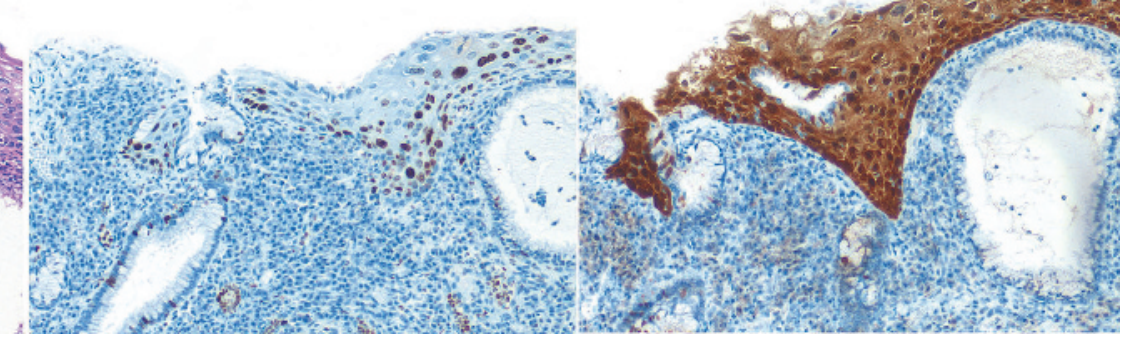

Figure 7 Low-grade squamous intraepithelial lesion (LG-SIL) versus high-grade squamous intraepithelial lesion (HG-SIL): (A) H\&E-stained section of a lesion with morphological features bordering on cervical intraepithelial neoplasia 1 (CIN1) and CIN2; and (B) Ki-67 immunohistochemistry exhibiting

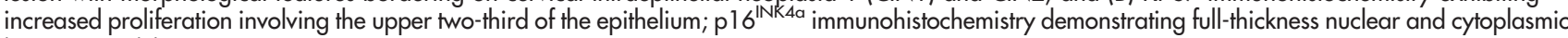
immunoreactivity

www.jclinpath.com 

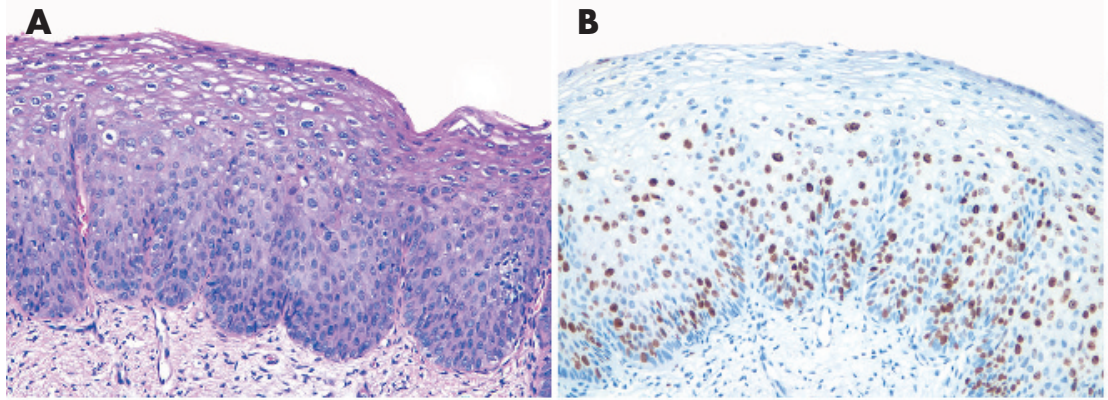

C

Figure 8 Low-grade squamous intraepithelial lesion (LG-SIL) (CIN 1): (A) H\&E-stained section exhibiting marked human papillomavirus-associated koilocytic changes; (B) Ki-67 immunohistochemistry showing positivity restricted to the lower half of the epithelium; and (C) negative p16 $6^{\text {INK4a }}$ immunohistochemistry.

the hypothesis that HPV DNA integration into the host DNA results in disruption of the E1/E2 open reading frames with loss of the E2-controlled regulation of E6 and E7. ${ }^{12}$

The direct relationship between the grade of cervical dysplasia and the intensity of $\mathrm{pl}^{\mathrm{INK} 4 \mathrm{a}}$ staining has been well established in recent years. However, significant variability remains in the reported percentages of CIN grades that are immunoreactive for $\mathrm{pl}^{\mathrm{INK} 4 \mathrm{a}}$. Most studies report negative to minimal pl6 ${ }^{\mathrm{INK} 4 \mathrm{a}}$ staining in normal or reactive cervical biopsy specimens, with the greatest variability in the number of low-grade CIN lesions that are $\mathrm{pl}^{\mathrm{INK} 4 \mathrm{a}}$ positive. Although most studies report nearly $90-100 \%$ pl $66^{\mathrm{INK} 4 \mathrm{a}}$ positivity in high-grade CIN lesions, ${ }^{19-21} 243233$ some investigators report up to $15-30 \%$ of high-grade CIN that are negative for $\mathrm{pl}^{\mathrm{INK} 4 \mathrm{a}} .^{18}{ }^{34}$ Accordingly, while a specific indicator of CIN, a negative result for $\mathrm{p} 16^{\text {INK4a }}$ does not exclude the presence of CIN. The pl6 ${ }^{\text {INK4a }}$ immunohistochemistry therefore plays an adjunctive/supportive role in conjunction with the morphological interpretation. Improvement in the heterogeneity of p16 $6^{\text {INK4a }}$ immunoexpression could come from: (1) more uniform morphological categorisation of CIN; (2) standardisation in the scoring of $\mathrm{pl}^{\text {INK4a }}$ immunoexpression (positive vs negative, nuclear and/or cytoplasmic staining, distribution within the epithelium); and (3) standardisation of the antibody clone used.

Patterns of $\mathrm{p} 16^{\mathrm{INK} 4 a}$ immunostaining LG-SIL (CIN 1) shows a characteristic focal to diffuse staining of $\mathrm{pl}^{\mathrm{INK} 4 \mathrm{a}}$ within the lower one-third to half of the epithelium. $^{20}$ HG-SIL (CIN 2 and 3) typically shows intense, diffuse staining for $\mathrm{pl} 6^{\mathrm{INK} 4 \mathrm{a}}$ in the upper two-thirds to full-thickness of the epithelium (figs $4 \mathrm{~F}$ and $5 \mathrm{C}$ ). Heterogenous staining has been reported in CIN 1 lesions with a range of positive LG-SIL cases ranging from 31\% to $100 \% .{ }^{18} 203334$ This heterogeneity may reflect the varying percentage of CIN 1 lesions harbouring HR HPV virus. Despite these differences, we reserve the role of p16 ${ }^{\text {INK4a }}$ immunohistochemistry as a potential marker of dysplasia in difficult lesions (eg, CIN l vs atypical squamous metaplasia), with the caveat that negative results does not exclude dysplasia. Nevertheless, heterogeneous $\mathrm{pl}^{\mathrm{INK} 4 \mathrm{a}}$ immunoreactivity has been reported in CIN 1 lesions, and its usefulness in this setting is not well established Heterogeneity in immunostaining may also represent a difference in potential aggressiveness among low-grade lesions, perhaps reflecting the different physical states of the virus (ie, episomal vs integrated). It is possible that the lowgrade lesions exhibiting higher levels of pl6 $6^{\text {INK4a }}$ immunoexpression could show a greater proportion of integrated HPV and may therefore be a useful potential marker of LG-SILs at risk of progression. Similarly, the dysplastic foci that are negative for p16 ${ }^{\text {INK4a }}$ (fig 8) may represent lesions that are in the process of regression with a potential for clearance of the virus. Naturally, there is significant difficulty in the interpretation of an evolving process through a single static picture, emphasising the role of multiple adjunctive modalities in the diagnosis of CIN.

\section{Use of HPV in situ hybridisation}

In situ hybridisation (ISH) can detect HPV within a morphological context and has been used as an indicator of HPV physical status within the host cell. HPV infection is initiated with episomal virus. This state is characterised by a diffuse ISH signal, correlating with the productive phenotype of HPV and the koilocytic changes seen in low-grade cervical dysplasia. ${ }^{7}$ HPV DNA integration has been found to be a good marker of high-grade lesions and invasive cervical carcinoma, and this event is characterised by a nuclear dot-like, or punctate, ISH signal. ${ }^{93}$ In a study of ISH signal patterns obtained using a highly sensitive tyramide-based ISH assay of 22 CIN 2/3 lesions and $26 \mathrm{CIN} 1$ lesions, all CIN 2/3 lesions (100\%) exhibited basal punctate signals (ie, integrated virus; fig 9). ${ }^{9}$ In contrast, only 5 of 26 (25\%) CIN 1 lesions exhibited basal punctate signals, the majority of which were associated with HR HPV types 16 and $18 .{ }^{9}$ It has been widely hypothesised that acquisition of integrated virus (especially in the basal cell layer) is critical for the development of a high-grade lesion through disruption of the HPV E1/E2 open reading frames. ${ }^{912}$ Examination of the physical status of the virus within a morphological/histopathological context is a valuable adjunct to the diagnosis of CIN. Indeed, evidence of viral integration through ISH (ie, punctate signal pattern) could prove to be a potential predictor of aggressive behaviour in these lesions and deserves further study.

\section{Use of proliferation marker $\mathrm{Ki}-67$}

The Ki-67 antigen detects cells in all active phases of the cell cycle and has been used as an indicator of CIN. Expressed normally in the parabasal cells of mature squamous epithelium, qualitative evaluation of Ki-67 cells involving the upper two-third of the epithelium has been reported to have improved specificity in detecting CIN (fig 2B, D). ${ }^{19} 213536$ As Ki-67 shows only sporadic focal staining of the basal/parabasal cells in atrophic biopsies (fig 6B), it is extremely helpful in distinguishing between cervical atrophy and high-grade dysplasia. Although being a sensitive marker of cervical neoplasia, the increased proliferation seen on Ki-67 must be interpreted with caution while examining reactive, inflammatory lesions. Maloriented specimens may also present difficulties in interpretation with denuded superficial aspects of the squamous epithelium, or if the basal and parabasal zones are tangentially sectioned. In addition, hormonal influences can effect Ki-67 staining in cervical epithelial cells with increased positivity in parabasal cells during the 


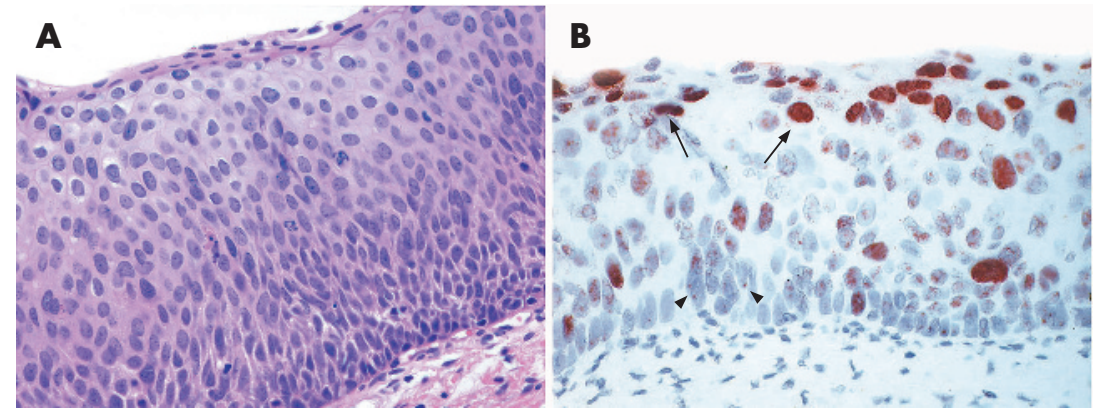

Figure 9 High-grade squamous intraepithelial lesion (HG-SIL) (CIN 3): (A) H\&E-stained section and (B) human papillomavirus in situ hybridisation exhibiting basal punctate signal (arrowheads) and diffuse signals towards the superficial aspect of the epithelium (black arrows).

luteal phase of the menstrual cycle and during pregnancy. ${ }^{37} 38$

A detailed discussion of the sensitivity and specificity of surrogate markers Ki-67 and $\mathrm{pl6}^{\mathrm{INK4a}}$ in the diagnosis of SIL, whether used in combination or alone, is deferred to a more extensive review elsewhere. ${ }^{39}$ Although a highly specific marker of HG-SIL, focal immunostaining for pl6 ${ }^{\mathrm{INK} 4 \mathrm{a}}$ has been reported in reactive and metaplastic lesions of the cervix, leading to the potential for false positives. In contrast, Ki-67 is a sensitive marker of HG-SIL, but lacks significant specificity, especially in the setting of inflammatory, reactive lesions. When resorting to ancillary studies in difficult cervical lesions, we always use both immunomarkers in conjunction with the morphological impression. Of the approximately 1000 colposcopically guided cervical biopsies we examined in 2005, we used ancillary studies (p16 $6^{\mathrm{INK} 4 \mathrm{a}}$ and Ki-67 immunohistochemistry) in approximately $10 \%$ of cases.

\section{SUMMARY}

Morphological analysis remains the "gold standard" in the diagnosis and grading of CIN; however, recently, immunohistochemical markers such as $\mathrm{pl}^{\mathrm{INK} 4 \mathrm{a}}$ and Ki-67 have emerged as helpful adjuncts to the diagnosis. They have proven beneficial in distinguishing high-grade cervical dysplasia from its benign mimics such as cervical atrophy, immature squamous metaplasia and reactive inflammatory lesions. As no ancillary test attains 100\% sensitivity or specificity, we advocate the combined use of $\mathrm{pl}^{\mathrm{INK} 4 \mathrm{a}}$ and Ki-67 in lesions that are morphologically suspicious for CIN. The utility of these markers in CIN screening has not been established. Their use in conjunction with possible ISH for HPV (episomal vs integrated signal patterns) has been shown to improve diagnostic accuracy and significantly reduce interobserver variability. ${ }^{31}$ Further large-scale studies are needed to examine the clinical usefulness of pl6 ${ }^{\text {INK4a }}$ immunostaining and HPV ISH

\section{Take-home messages}

- Morphological analysis remains the "gold standard" in the grading of cervical intraepithelial neoplasia $(\mathrm{CIN})$, but significant intraobserver and interobserver variability in the diagnosis of CIN still exists.

- Potential benign mimics of highgrade cervical dysplasia (CIN 2 and 3) include basal-cell hyperplasia, immature squamous metaplasia, reactive/inflammatory lesions and squamous atrophy.

- Surrogate markers such as immunohistochemistry for $\mathrm{pl}^{\mathrm{INK} 4 \mathrm{a}}$ and $\mathrm{Ki}-67$ have been shown to improve diagnostic accuracy and reduce interobserver variability.

- We support the combined use of pl6 ${ }^{\text {INK4a }}$ and Ki-67 immunohistochemistry in lesions that are morphologically suspicious for CIN.

signal patterns, alone or in combination, in identifying CIN 1 lesions at higher risk of progression.

\section{ACKNOWLEDGEMENTS}

We thank Dr Mark F Evans for his invaluable expertise and assistance in the HPV-ISH projects.

J Clin Pathol 2007;60:449-455. doi: 10.1136/icp.2005.036426

\section{Authors' affiliations}

Alexandra N Kalof, Kumarasen Cooper, University of Vermont, Burlington, Vermont, USA

Correspondence to: Dr A N Kalof, Department of Pathology, University of Vermont, Fletcher Allen
Health Care, ACC-E2-108, 111 Colchester Avenue, Burlington, VT 05401, USA; alexandra. kalof@vtmednet.org

Accepted 28 September 2006

Published Online First 17 October 2006

Competing interests: None declared.

\section{REFERENCES}

1 Schiffman MH, Baver HM, Hoover RN, et al Epidemiologic evidence showing that human papillomavirus infection causes most cervical intraepithelial neoplasia. J Natl Cancer Inst 1993;85:958-64.

2 Walboomers JM, Jacobs MV, Manos MM, et al Human papillomavirus is a necessary cause of invasive cervical cancer worldwide. J Pathol 1999; 189:12-19.

3 Heatley MK. How should we grade CIN? Histopathology 2002;40:377-90.

4 McCluggage WG, Bharucha $H$, Caughley $L M$, et al. Interobserver variation in the reporting of cervical colposcopic biopsy specimens: comparison of grading systems. J Clin Pathol 1996;49:833-5.

5 Stoler MH, Schiffman M. Interobserver reproducibility of cervical cytologic and histologic interpretations: realistic estimates from the ASCUSLSIL Triage Study. JAMA 2001;285:1500-5.

6 Mount S, Evans M, Wong C, et al. Human papillomavirus-induced lesions of the cervix. Pathol Case Rev 2003;8:145-51.

7 Cooper K, Evans M, Mount S. Biology and evolution of cervical squamous intraepithelial lesions: a hypothesis with diagnostic prognostic implications. Adv Anat Pathol 2003;10:200-3.

8 Ostor AG. Natural history of cervical intraepithelia neoplasia: a critical review. Int J Gynecol Pathol 1993; 12:186-92.

9 Evans MF, Mount SL, Beatty BG, et al. Biotinyltyramide-based in situ hybridization signal patterns distinguish human papillomavirus type and grade of cervical intraepithelial neoplasia. Mod Pathol 2002; 15:1339-47.

10 Ho GY, Burk RD, Klein S, et al. Persistent genital human papillomavirus infection as a risk factor for persistent cervical dysplasia. J Natl Cancer Inst 1995;87:1365-71

11 Jeon S, Allen-Hoffmann BL, Lambert PF. Integration of human papillomavirus type 16 into the human genome correlates with a selective growth advantage of cells. J Virol 1995;69:2989-97.

12 Peitsaro $\mathbf{P}$, Johansson B, Syrjanen S. Integrated human papillomavirus type 16 is frequently found in cervical cancer precursors as demonstrated by a novel quantitative real-time PCR technique. J Clin Microbiol 2002;40:886-91

13 McCluggage WG, Walsh MY, Thornton CM, et al Inter-and intra-observer variation in the histopathological reporting of cervical squamous intraepithelial lesions using a modified Bethesda grading system. Br J Obstet Gynaecol 1998;105:206-10

14 Anderson MC, Robboy SJ, Russell P, et al. The cervix: benign and non-neoplastic conditions. In: Robboy SJ, Anderson MC, Russell P, eds. Pathology of the female reproductive tract. London: Churchill Livingstone, 2002:105-35

15 Robboy SJ, Anderson MC, Morse A, et al. Cervica precancer (intraepithelial neoplasia). In: Robboy SJ, Anderson MC, Russell P, eds. Pathology of the female reproductive tract. London: Churchill Livingstone, 2002:165-93

16 Wilson GE. How should we grade CIN? Histopathology 2002;40:380-5.

17 Leung KM, Chan WY, Hui PK. Invasive squamous cell carcinoma and cervical intraepithelial neoplasia III of uterine cervix. Morphologic differences other than stromal invasion. Am J Clin Pathol 1994;101:508-13.

18 Agoff SN, Lin P, Morihara J, et al. pl6 (INK4a) expression correlates with degree of cervical neoplasia: a comparison with $\mathrm{Ki}-67$ expression and detection of high-risk HPV types. Mod Pathol 2003; 16:665-73. 
19 Keating JT, Cviko A, Riethdorf S, et al. Ki-67, cyclin $\mathrm{E}$, and p16INK4 are complimentary surrogate
biomarkers for human papilloma virus-related cervical neoplasia. Am J Surg Pathol 2001;25:884-91

20 Klaes R, Friedrich T, Spitkovsky D, et al. Overexpression of p16 (INK4A) as a specific marker for dysplastic and neoplastic epithelial cells of the cervix uteri. Int J Cancer $2001 ; 92: 276-84$.

21 Lorenzato M, Caudroy S, Bronner C, et al. Cell cycle and/or proliferation markers: what is the best method to discriminate cervical high-grade lesions? Hum Pathol 2005;36:1101-7.

22 Murphy N, Ring M, Heffron CC, et al. pl6INK4A, CDC6, and MCM5: predictive biomarkers in cervical preinvasive neoplasia and cervical cancer. J Clin Pathol 2005;58:525-34.

23 Sano T, Oyama T, Kashiwabara K, et al. Expression status of pl 6 protein is associated with human papillomavirus oncogenic potential in cervical and genital lesions. Am J Pathol 1998;153:1741-8.

24 Wang SS, Trunk M, Schiffman M, et al. Validation of pl6INK4a as a marker of oncogenic human papillomavirus infection in cervical biopsies from a population-based cohort in Costa Rica. Cancer Epidemiol Biomarkers Prev 2004; 13:1355-60.

25 Chakravarti A, Heydon K, Wu CL, et al. Loss of p16 expression is of prognostic significance in locally advanced prostate cancer: an analysis from the Radiation Therapy Oncology Group protocol 8610. J Clin Oncol 2003:21:3328-34.
26 Feakins RM, Nickols CD, Bidd $\mathrm{H}$, et al. Abnormal expression of $\mathrm{pRb}, \mathrm{pl}$, and cyclin D1 in gastric adenocarcinoma and its lymph node metastases: relationship with pathological features and survival. Hum Pathol 2003;34:1276-82.

27 Kawaguchi K, Oda Y, Saito T, et al. Mechanisms of inactivation of the pl6INK4a gene in

leiomyosarcoma of soft tissue: decreased pl6 expression correlates with promoter methylation and poor prognosis. J Pathol 2003;201:487-95.

28 Leversha MA, Fielding $P$, Watson S, et al. Expression of p53, pRB, and p16 in lung tumours: a validation study on tissue microarrays. J Pathol 2003;200:610-19

29 Makitie AA, MacMillan C, Ho J, et al. Loss of pl6 expression has prognostic significance in human nasopharyngeal carcinoma. Clin Cancer Res 2003;9:2177-84.

30 Mihic-Probst D, Saremaslani P, Komminoth P, et al. Immunostaining for the tumour suppressor gene pl6 product is a useful marker to differentiate melanoma metastasis from lymph-node nevus. Virchows Arch 2003;443:745-51.

31 Klaes R, Benner A, Friedrich T, et al. pl6INK4a immunohistochemistry improves interobserver agreement in the diagnosis of cervical intraepithelial neoplasia. Am J Surg Pathol 2002;26:1389-99.

32 Benevolo M, Mottolese M, Marandino F, et al. Immunohistochemical expression of pl6 (INK4a) is predictive of HR-HPV infection in cervical low-grade lesions. Mod Pathol 2006;19:384-91.
33 Kalof AN Evans MF Simmons-Arnold $L$, et al. pl6INK4A immunoexpression and HPV in situ hybridization signal patterns: potential markers of high-grade cervical intraepithelial neoplasia. Am J Surg Pathol 2005;29:674-9.

34 Branca M, Ciotti M, Santini D, et al. pl6 (INK4A) expression is related to grade of cin and high-risk human papillomavirus but does not predict virus clearance after conization or disease outcome. Int J Gynecol Pathol 2004;23:354-65

35 Kruse AJ, Baak JP, Helliesen T, et al. Evaluation of MIB-1-positive cell clusters as a diagnostic marker for cervical intraepithelial neoplasia. Am J Surg Pathol 2002;26:1501-7.

36 McCluggage WG, Buhidma $M$, Tang $L$, et al. Monoclonal antibody MIB1 in the assessment of cervical squamous intraepithelial lesions. Int J Gynecol Pathol 1996;15:131-6.

37 Keating JT, Ince T, Crum CP. Surrogate biomarkers of HPV infection in cervical neoplasia screening and diagnosis. Adv Anat Pathol 2001:8:83-92.

38 Konishi I, Fujii S, Nonogaki $\mathrm{H}$, et al. Immunohistochemical analysis of estrogen receptors, progesterone receptors, $\mathrm{Ki}-67$ antigen and human papillomavirus DNA in normal and neoplastic epithelium of the uterine cervix. Cancer 1991;68:1340-50.

39 Kalof AN, Cooper K. pl6INK4a immunoexpression: surrogate marker of high-risk HPV and high-grade cervical intraepithelial neoplasia. Adv Anat Pathol 2006;13:190-4. 